\title{
Sublogarithmic behaviour of the entanglement entropy in fermionic chains
}

\author{
Filiberto Ares*坾 \\ Departamento de Física Teórica, Universidad de Zaragoza, 50009 Zaragoza, Spain and \\ International Institute of Physics, UFRN, 59078-970, Natal, RN, Brazil \\ José G. Esteve尹 and Fernando Falcetd\$ \\ Departamento de Física Teórica, Universidad de Zaragoza, 50009 Zaragoza, Spain and \\ Instituto de Biocomputación y Física de Sistemas Complejos (BIFI), 50009 Zaragoza, Spain
}

\section{Zoltán Zimborás}

Theoretical Physics Department, Wigner Research Centre for Physics,

$$
\text { P.O.Box } 49 \text { H-1525, Budapest, Hungary }
$$

MTA-BME Lendület Quantum Information Theory Research Group, Budapest, Hungary and Mathematical Institute, Budapest University of Technology and Economics,

$$
\text { P.O.Box } 91 \text { H-1111, Budapest, Hungary }
$$

In this paper, we discuss the possibility of unexplored behaviours for the entanglement entropy in extended quantum systems. Namely, we study the Rényi entanglement entropy for the ground state of long-range Kitaev chains with slow decaying couplings. We obtain that, under some circumstances, the entropy grows sublogarithmically with the length of the subsystem. Our result is based on the asymptotic behaviour of a new class of Toeplitz determinants whose symbol does not lie within the application domain of the Strong Szegő Theorem or the Fisher-Hartwig conjecture.

\footnotetext{
* Corresponding author.

†Electronic address: fares@iip.ufrn.br

$\ddagger$ Electronic address: esteve@unizar.es

$\S$ Electronic address: falceto@unizar.es

ฯElectronic address: zimboras.zoltan@wigner.mta.hu
} 


\section{INTRODUCTION}

In 1973 Bekenstein suggested [1] and shortly afterwards Hawking proved [2] that the entropy of a black hole is proportional to the area of its events horizon, contrary to the usual thermal entropy, that is proportional to the volume of the system. Trying to better understand the Bekenstein-Hawking entropy, Sorkin and collaborators considered in the pioneer work [3] a real scalar field and computed the von Neumann entanglement entropy of the reduced density matrix of the ground state restricted to a region of the space. They found that this entropy is proportional to the boundary area that separates this region from the rest of the space; i.e. similarly to the black hole, the entropy follows an area law. The same property was also found later by Sredincki in [4]. This important result not only revealed that entanglement may play a role in the black hole physics, but also indicated that the area law may appear in other situations.

As in the example above, the entanglement entropy often follows an area law. It is commonly admitted that this occurs in the ground state of theories with mass gap and finiterange interactions. This feature was indeed proved by Hastings [5] for one-dimensional systems, in which the area law implies that the entanglement entropy saturates to a constant value in the large size limit. On the contrary, when the mass gap is zero, the area law is violated and the entanglement entropy typically grows with the logarithm of the length of the interval considered, its coefficient being proportional to the central charge of the underlying conformal field theory [6-8].

The presence of infinite-range interactions enriches the previous picture. For example, in the fermionic ladders studied by the authors in Ref. [9], the leading term of the ground state entanglement entropy is proportional to the length of the interval, i.e. a volume law. In Ref. [10], Ercolessi and collaborators introduced a long-range Kitaev chain with pairing couplings that decay with a power law. These systems may exhibit a logarithmic growth of the entropy even outside the critical points of the system [11]. Moreover, as was discovered in [12], the logarithmic term may be non universal and cannot be derived from a conformal field theory. Violations of the area law have been found also in systems without translational invariance where the couplings force the accumulation of singlet bonds at some points, or when there are random and inhomogeneous couplings [13 16]

A natural question is if the entanglement entropy can present other behaviours with 
the size of the subsystem, apart from the volume and the area laws or the logarithmic growth. There are some recent works that address this issue.

In Ref. [19], Movassagh and Shor introduced a translational invariant and local spin chain whose ground state entanglement entropy grows with the square root of the length of the interval. This result has been extended to other spin chains, see e.g. Refs. [20, 21]. In Refs. [22 24], it was found that, in quadratic fermionic chains with long-range couplings that lead to a fractal Fermi surface, the entanglement entropy also grows with a power of the length of the interval.

On the other hand, in Ref. [25] Bianchini et al. analyzed the entanglement entropy in non-unitary field theories and obtained that sublogarithmic terms may be present in the case of logarithmic conformal field theories, see also [26, 27]. Some numerical studies like [28] in Ising spin chains with long-range interactions also suggest the possibility of finding sublogarithmic terms in the entanglement entropy.

Motivated by these works, one can wonder if it is possible to engineer a fermionic chain with long-range couplings in which the entanglement entropy of the ground state may display a sublogarithmic growth.

One method to compute the entanglement entropy in quadratic fermionic chains is to express it in terms of the two-point correlation matrix. Then, if the chain is translational invariant, one can exploit the properties of Toeplitz and block Toeplitz determinants and find the asymptotic expansion of the entropy with the length of the subsystem.

The Strong Szegő Theorem and the Fisher-Hartwig conjecture for Toeplitz determinants, as well as their generalisations to the block Toeplitz case, are the two main results describing their asymptotic behaviour. According to these results, the entanglement entropy must grow linearly or logarithmically with the size of the subsystem, or tend to a constant value.

However, one should take into account that these theorems have a range of validity. The Strong Szegö Theorem only applies to Toeplitz matrices generated by a smooth enough, non-zero symbol. The Fisher-Hartwig conjecture extends the former to symbols with jump discontinuities and/or zeros.

Therefore, the fermionic chains in which the entanglement entropy behaves in a different way would correspond to symbols that break the hypothesis of the Strong Szegö Theorem and the Fisher-Hartwig conjecture. For example, a continuous, non-zero symbol 
(then the Fisher-Hartwig conjecture does not apply) but non smooth enough according to the conditions imposed by the Strong Szegö Theorem.

Interestingly enough, to our knowledge, the results in the literature do not cover the asymptotic behaviour of Toeplitz determinants for a symbol with those features.

In this paper, we shall propose a conjecture for the asymptotic behaviour of a family of continuous symbols whose derivative diverges at a single point, generalising the result of Fisher and Hartwig. According to our conjecture, the leading term in the logarithm of their Toeplitz determinant is sublogarithmic in its dimension. The numerical checks will leave little doubt about the correctness of this result. We will apply this result to the calculation of the Rényi entanglement entropy for the ground state of long-range Kitaev chains with pairing couplings that decay slowly with the distance, obtaining that it grows sublogarithmically with the length of the interval. To the best of our knowledge, this property has not been reported before and constitutes the main result of this work.

The paper is organized as follows. In Section [I], we present the physical systems of interest, the long-range Kitaev chains, reviewing the known results for the ground state entanglement entropy. In Section [II], we discuss the Strong Szegő Theorem and the FisherHartwig conjecture for Toeplitz determinants and we consider a family of symbols for which these results are not valid. We propose for this family of symbols a new asymptotic regime that leads to the sublogarithmic growth of the logarithm of the determinant with its dimension. In Section IV, using this result, we extract analytically the leading term of the Rényi entanglement entropy in the ground state of long-range Kitaev chains with slow decaying pairing couplings. We finish in Section $\mathrm{V}$ with the conclusions. We have also included an Appendix where we describe several results on Toeplitz and block Toeplitz determinants that could be useful to the reader.

\section{LONG-RANGE KITAEV CHAINS}

In this paper we are interested in long range Kitaev chains. Its Hamiltonian is given by

$$
H=\sum_{m=1}^{M}\left(a_{m}^{\dagger} a_{m+1}+a_{m+1}^{\dagger} a_{m}+h a_{m}^{\dagger} a_{m}\right)+\sum_{m=1}^{M} \sum_{0<|l| \leq M / 2} \gamma_{l}\left(a_{m}^{\dagger} a_{m+l}^{\dagger}-a_{m} a_{m+l}\right)-\frac{M h}{2}
$$


where $a_{m}^{\dagger}, a_{m}$ are the fermionic creation and annihilation operators fulfilling the canonical anticommutation relations

$$
\left\{a_{m}^{\dagger}, a_{n}\right\}=\delta_{m n}, \quad\left\{a_{m}^{\dagger}, a_{n}^{\dagger}\right\}=\left\{a_{m}, a_{n}\right\}=0, \quad m, n=1, \ldots, M
$$

and we consider periodic boundary conditions, $a_{M+m}=a_{m}$. The hopping terms only couple nearest neighbours but the pairing $\gamma_{l}$ extends to the whole chain and satisfies $\gamma_{l}=-\gamma_{-l}$. In order to keep certain sense of locality we shall assume that they attenuate at infinity, i.e. $\gamma_{l} \rightarrow 0$ when $l \rightarrow \pm \infty$.

This is a free, translational invariant system whose elementary excitations are characterized by its momentum $k$. The dispersion relation tells us the energy associated to each mode. In the thermodynamic limit and denoting by $\theta=2 \pi k / M$ it is

$$
\omega(\theta)=\sqrt{(h+2 \cos \theta)^{2}+|G(\theta)|^{2}},
$$

where

$$
G(\theta)=\sum_{l=1}^{\infty}\left(\mathrm{e}^{\mathrm{i} \theta l}-\mathrm{e}^{-\mathrm{i} \theta l}\right) \gamma_{l}
$$

An example of this system that has been most thoroughly studied corresponds to

$$
\gamma_{l}=\operatorname{sign}(l)|l|^{-\delta}, \quad \delta>0, \quad l \neq 0 .
$$

Therefore, the Hamiltonian takes the form

$H_{\mathrm{LRK}}=\sum_{m=1}^{M}\left(a_{m}^{\dagger} a_{m+1}+a_{m+1}^{\dagger} a_{m}+h a_{m}^{\dagger} a_{m}\right)+\sum_{m=1}^{M} \sum_{0<|l| \leq M / 2} l|l|^{-\delta-1}\left(a_{m}^{\dagger} a_{m+l}^{\dagger}-a_{m} a_{m+l}\right)-\frac{M h}{2}$.

This model was introduced a few years ago in the pioneering paper [10].

Since then it has been the object of an intense study. It is very useful to analyse the effects of long-range interactions. Here we shall restrict to the entanglement entropy but different authors have used the model to investigate the form of the correlations [10, 29, 30], the breaking of conformal symmetry [31, the propagation of information [32], the behaviour out of equilibrium [33] or the occurrence and structure of topological phases [34-39].

As we just mentioned, in this paper we will be interested in the Rényi entanglement entropy for a subsystem $X$ in the ground state $|\mathrm{GS}\rangle$ of the chain. It is defined by

$$
S_{\alpha, X}=\frac{1}{1-\alpha} \log \operatorname{Tr}_{X}\left(\rho_{X}^{\alpha}\right)
$$


where $\rho_{X}=\operatorname{Tr}_{Y}|\mathrm{GS}\rangle\langle\mathrm{GS}|$ is the reduced density matrix obtained by tracing out, in the pure ground state, the degrees of freedom of the subsystem $Y$ complementary to $X$. In the limit $\alpha \rightarrow 1$ we recover the von Neumann entropy.

In the original paper [10] the authors compute numerically the scaling behaviour of the entanglement entropy as a function of the dumping exponent $\delta$ for $\delta \neq 1$. The interest of the model resides in the fact that it violates the standard connection with conformal field theories.

In fact, according to the latter, for a one dimensional, critical, local theory the Rényi entanglement entropy of a subsystem $X$ grows logarithmically with its size $|X|$ with a coefficient proportional to the central charge of the theory c. Namely

$$
S_{\alpha, X} \sim \frac{\alpha+1}{6 \alpha} \mathrm{clog}|X|
$$

Also, in a free theory like the one at hand, the central charge is the number of massless bosonic particles or one half of the fermionic ones. In concrete terms, in our fermionic spinless system, the central charge corresponds to one half of the number of zeros in the dispersion relation.

In order to apply the previous results to (5) we must compute the dispersion relation, i.e. the energy of the single particle eigenstate of momentum $k$. Particularizing (3) to the case (5) we have that, in the thermodynamic limit,

$$
\omega_{\mathrm{LRK}}(\theta)=\sqrt{(h+2 \cos \theta)^{2}+\left|G_{\mathrm{LRK}}(\theta)\right|^{2}},
$$

where

$$
G_{\mathrm{LRK}}(\theta)=\operatorname{Li}_{\delta}\left(\mathrm{e}^{\mathrm{i} \theta}\right)-\mathrm{Li}_{\delta}\left(\mathrm{e}^{-\mathrm{i} \theta}\right)
$$

and $\mathrm{Li}_{\delta}$ stands for the polylogarithm of order $\delta$ [40],

$$
\operatorname{Li}_{\delta}(z)=\sum_{l=1}^{\infty} \frac{z^{l}}{l^{\delta}} .
$$

This is analytic for $|z|<1$ and extends, for $\delta>0$, to a multivalued function in the whole complex plane excluding, in some cases, $z=1$. In fact, $\mathrm{Li}_{\delta}$ has a finite limit at $z=1$ for $\delta>1$ while it diverges at this point for $\delta \leq 1$. For $\delta=1$ the polylogarithm function reduces to the logarithm, namely $\operatorname{Li}_{1}(z)=-\log (1-z)$.

As we mentioned before, massless excitations are associated to the zeros of the dispersion relation (7). It only vanishes for $h=2$ at $\theta_{F}=\pi$ or for $h=-2$ and $\theta_{F}=0$ [? ]. 
Therefore, according to the general theory (for local systems) one could expect that the entanglement entropy in the vacuum state goes to a constant for $h \neq \pm 2$ in the large $|X|$ limit and it behaves like

$$
S_{\alpha, X} \sim \frac{\alpha+1}{6 \alpha} \frac{1}{2} \log |X|
$$

for $h= \pm 2$.

In [11] and [12], using some novel results derived by the authors on the asymptotic behaviour for block Toeplitz determinants with discontinuous symbols, we have been able to compute the large $|X|$ limit of the entanglement entropy for the vacuum state of the long range Kitaev chain (5). The results, that we will outline below, do not follow the previous guess and rather confirm the numerical research of [10].

We first consider the case $\delta \neq 1$ that was the subject of refs. [10, 11]. In this case we can show that the entanglement entropy follows the general form

$$
S_{\alpha, X} \sim \frac{\alpha+1}{6 \alpha} \mathrm{clog}|X|
$$

but contrary to what is expected from conformal field theory (c $=1 / 2$ for $h= \pm 2$ and $\mathrm{c}=0$ otherwise) we have the following:

$$
c= \begin{cases}0 & \text { for } \delta>1 \text { and } h \neq \pm 2 \\ 1 / 2 & \text { for } \delta<1 \text { and } h \neq 2 \text { or } \delta>1 \text { and } h= \pm 2 \\ 1 & \text { for } \delta<1 \text { and } h=2 .\end{cases}
$$

Of course, the explanation for this deviation from the predicted result lays in the fact that the theory is non local (it has non zero coupling at all distances) and therefore the premises of the conformal field theory prediction are not met.

Anyhow, a more interesting result is obtained for $\delta=1$. This case is technically more complex and it has been discussed thoroughly in [12]. In this situation the Rényi entanglement entropy does not even follow the general expression (6), but rather it behaves like

$$
S_{\alpha, X}=B_{\alpha} \log |X|+O(1)
$$

where

$$
B_{\alpha}=\frac{2}{\pi^{2}} \int_{\cos \xi}^{1} \frac{\mathrm{d} f_{\alpha}(\lambda)}{\mathrm{d} \lambda} \log \frac{\sqrt{1-\lambda^{2}}}{\sqrt{\lambda^{2}-\cos ^{2} \xi}+\sin \xi} \mathrm{d} \lambda
$$


with

$$
\cos \xi=\frac{h+2}{\sqrt{(h+2)^{2}+\pi^{2}}}, \quad \sin \xi=\frac{\pi}{\sqrt{(h+2)^{2}+\pi^{2}}} .
$$

and

$$
f_{\alpha}(x)=\frac{1}{1-\alpha} \log \left[\left(\frac{1+x}{2}\right)^{\alpha}+\left(\frac{1-x}{2}\right)^{\alpha}\right] .
$$

Therefore, the coefficient of the logarithmic term in the asymptotic behaviour of the Rényi entanglement entropy does not depend on $\alpha$ as in (6), loosing any connection with conformal field theory. Similar type of results can be found in [17, 18].

The goal of the next sections is to go even further in exploring possible anomalous asymptotic behaviours for the entanglement entropy. In fact, we will show that it is possible to choose the pairing $\gamma_{l}$ in (1) so that the entropy grows like a fractional power of the logarithm of the size of the subsystem $X$.

\section{SUBLOGARITHMIC BEHAVIOUR OF TOEPLITZ DETERMINANTS}

As it has been discussed previously in a number of papers, see e.g. [9, 11, 12, 41 45], and will be recalled in the next section, the entanglement entropy of the free fermionic chain can be derived from the computation of a Toeplitz determinant. Therefore, before addressing the problem mentioned at the end of the previous section we have to establish some new results on the asymptotic behaviour of Toeplitz determinants in the following paragraphs.

The history of Toeplitz operators and Toeplitz determinants is an interesting example of crossbreading between physics and mathematics. It originated from a branch of pure abstract mathematics that very soon proved to be essential for physics: the Hilbert space.

Toeplitz operators were introduced in 1907 by Otto Toeplitz [46], at Göttingen at that time, to provide concrete examples of the abstract theory that David Hilbert was developping there. We will be interested in the asymptotic behaviour of Toeplitz determinants as defined below.

For a complex function $f$ on the unit circle $S^{1}$ with $f \in L^{1}\left(S^{1}\right)$, we shall denote by $T_{N}[f]$ the Toeplitz matrix with symbol $f$ and dimension $N \times N$. Its entries $\left(T_{N}[f]\right)_{n m}=f_{n-m}$ are given by the Fourier coefficients of the function $f$,

$$
f_{k}=\frac{1}{2 \pi} \int_{-\pi}^{\pi} f(\theta) \mathrm{e}^{\mathrm{i} \theta k} \mathrm{~d} \theta
$$


The first result on the large $N$ limit of its determinant $D_{N}[f] \equiv \operatorname{det} T_{N}[f]$ was conjectured by Polya [47] and proved by Szegö in [48]. It states the following:

First Szegő theorem (1915)

Assume that $f \in L^{1}\left(S^{1}\right)$ is not vanishing and has zero winding around zero, then its Toeplitz determinant verifies

$$
\log D_{N}[f]=\frac{N}{2 \pi} \int_{-\pi}^{\pi} \log f(\theta) \mathrm{d} \theta+o(N)
$$

This result was not improved until the early 50's motivated by Kauffman and Onsager discoveries in statistical physics [49]. Actually in 1949 they established that the magnetization of the classical Ising model in two dimensions below critical temperature could be obtained from the computation of a particular Toeplitz determinant [50]. But Szegő's theorem on the asymptotic limit of the determinant gives a trivial result in their case, and the answer was in the $o(N)$ correction of 10 which is not determined by the theorem.

Using an alternative derivation of the magnetization, Onsager apparently formulated a conjecture for the subleading term, but he did not publish neither the conjecture nor the result on the magnetization.

While Onsager was trying to prove his guess, Szegő was aware of the problem and soon after he came out with the proof. Today it is known as the Strong Szegö Theorem (1952) that can be stated as follows [51, 52]:

Assume that the symbol $f \in L^{1}\left(S^{1}\right)$ is not vanishing, has zero winding number around zero and it is well-behaved in the sense that its Fourier modes $f_{k}$ satisfy

$$
\sum_{k=-\infty}^{\infty}\left|f_{k}\right|+\sum_{k=-\infty}^{\infty}|k|\left|f_{k}\right|^{2}<\infty
$$

then the correction in (10) is finite in the limit $N \rightarrow \infty$,

$$
\log D_{N}[f]=\frac{N}{2 \pi} \int_{-\pi}^{\pi} \log f(\theta) \mathrm{d} \theta+\sum_{k=1}^{\infty} k s_{k} s_{-k}+o(1),
$$

where the $s_{k}$ 's in the sum are the Fourier modes of $\log f(\theta)$,

$$
s_{k}=\frac{1}{2 \pi} \int_{-\pi}^{\pi} \log f(\theta) \mathrm{e}^{\mathrm{i} \theta k} \mathrm{~d} \theta .
$$


A little later it became clear that statistical physicists needed to know the asymptotic behaviour for Toeplitz determinants with more general symbols. In fact as it was shown in 1963 by Lenard [53] the one particle density matrix of impenetrable bosons can be expressed as a Toeplitz determinant with zeros in the symbol which are excluded in Szegö's theorems. Also in the Ising model at critical temperature the magnetization derives from a Toeplitz determinant for a symbol with jump discontinuities. In $1966 \mathrm{Wu}$ [54] was able to derive the asymptotic behaviour of these determinants.

Putting these previous results together Fisher and Hartwig arrived in 1968 at their remarkable conjecture published in [55] and proven in full generality in 1979 by Basor in [56].

Here we shall discuss a particular version of the conjecture that only allows for discontinuities of the symbol (not zeros) as these are the kind of Toeplitz determinants that appear in connection with the entanglement entropy.

Accordingly, consider that the symbol $f$ has jump discontinuities at $\theta_{1}, \ldots, \theta_{R}$. The Fisher-Hartwig conjecture for this case says that the discontinuities contribute to $\log D_{N}[f]$ with a logarithmic term, whose coefficient only depends on the lateral limits $f_{r}^{ \pm}=\lim _{\theta \rightarrow \theta_{r}^{ \pm}} f(\theta), r=1, \ldots, R$. That is,

$$
\log D_{N}[f]=N s_{0}+\frac{\log N}{4 \pi^{2}} \sum_{r=1}^{R}\left(\log \frac{f_{r}^{+}}{f_{r}^{-}}\right)^{2}+\log E[f]+o(1) .
$$

The reasoning followed by Fisher and Hartwig in [55] to deduce the expansion (13) is as follows.

For simplicity, and as they precisely did, we take the symbol

$$
f^{(0)}(\theta)=\mathrm{e}^{\beta(\theta-\pi \operatorname{sign}(\theta))}, \quad \theta \in[-\pi, \pi)
$$

that only has a discontinuity at $\theta=0$.

The Fourier coefficients of $\log f^{(0)}$ are

$$
s_{0}^{(0)}=0, \quad \text { and } \quad s_{k}^{(0)}=\frac{\beta}{\mathrm{i} k}, \quad \text { for } \quad k \neq 0 .
$$

Then if we apply to this symbol the Strong Szegö Theorem 12 we obtain the Harmonic series

$$
\log D_{N}\left[f^{(0)}\right]=N s_{0}^{(0)}+\sum_{k=1}^{\infty} k s_{k}^{(0)} s_{-k}^{(0)}+o(1)=\sum_{k=1}^{\infty} \frac{\beta^{2}}{k}+o(1)
$$


that diverges logarithmically.

Let us suppose that $\log D_{N}\left[f^{(0)}\right]$ can be obtained truncating the series $\sum_{k=1}^{\infty} k s_{k}^{(0)} s_{-k}^{(0)}$ at some $k=\left\lfloor N \Lambda_{0}\right\rfloor$, with $\Lambda_{0}$ a positive real number (here $\lfloor t\rfloor$ means integer part of $t$ ). Then we find

$$
\log D_{N}\left[f^{(0)}\right]=\sum_{k=1}^{\left\lfloor N \Lambda_{0}\right\rfloor} k s_{k}^{(0)} s_{-k}^{(0)}=\sum_{k=1}^{\left\lfloor N \Lambda_{0}\right\rfloor} \frac{\beta^{2}}{k}=\beta^{2} \log \left(N \Lambda_{0}\right)+\beta^{2} \gamma_{\mathrm{E}}+o(1),
$$

where $\gamma_{\mathrm{E}}$ is the Euler-Mascheroni constant.

Notice that this truncation gives precisely the Fisher-Hartwig expansion (13) for the symbol $f^{(0)}$. In fact, since $f^{(0)}(\theta)$ presents a single discontinuity $(R=1)$ with lateral limits $\mathrm{e}^{ \pm \beta \pi}$, the expression in $(13)$ particularises as

$$
\log D_{N}\left[f^{(0)}\right]=\beta^{2} \log N+\log E\left[f^{(0)}\right]+o(1)
$$

Comparing (16) with $(15)$ we can conclude that

$$
\log E\left[f^{(0)}\right]=\beta^{2}\left(\log \Lambda_{0}+\gamma_{\mathrm{E}}\right)
$$

Fisher and Hartwig were able to fix the constant term $E\left[f^{(0)}\right]$ and, therefore, the cutoff parameter $\Lambda_{0}$ because they realised that the Toeplitz matrix with symbol $f^{(0)}$ is also a Cauchy matrix [? ]. Using the properties of the determinants of Cauchy matrices they determined that

$$
E\left[f^{(0)}\right]=\mathrm{G}(1+\mathrm{i} \beta) \mathrm{G}(1-\mathrm{i} \beta)
$$

where $\mathrm{G}(z)$ is the Barnes G-function. Hence we have

$$
\log \Lambda_{0}=2 \beta^{-2} \log |\mathrm{G}(1+\mathrm{i} \beta)|-\gamma_{\mathrm{E}}
$$

The same reasoning can be applied to a general symbol with $R$ discontinuities. The asymptotic behaviour of its determinant predicted by (13) can be deduced from the Strong Szegö theorem 12 truncating the divergent terms in the series $\sum_{k=1}^{\infty} k s_{k} s_{-k}$.

We want to go one step further in this generalization process. For that consider the symbol

$$
\log f^{(\nu)}(\theta)=\beta \frac{\theta-\pi \operatorname{sign}(\theta)}{\left(-\log \frac{|\theta|}{2 \pi}\right)^{\nu}}, \quad \theta \in[-\pi, \pi)
$$

with $\nu \geq 0$. 
It is clear that for $\nu>0, f^{(\nu)}$ is continuous but not differentiable at $\theta=0$. The Fourier coefficients of $\log f^{(\nu)}$ are

$$
s_{k}^{(\nu)}=\frac{\beta}{\mathrm{i} k(\log |2 \pi k|)^{\nu}}\left[1+O\left(\frac{1}{\log |k|}\right)\right] .
$$

and, therefore, the series

$$
\sum_{k=1}^{\infty} k s_{k}^{(\nu)} s_{-k}^{(\nu)}
$$

diverges for $\nu \leq 1 / 2$.

Now we proceed in a way similar to that of Fisher and Hartwig and establish the following conjecture

$$
\log D_{N}\left[f^{(\nu)}\right]=N s_{0}^{(\nu)}+\sum_{k=1}^{\left\lfloor N \Lambda_{\nu}\right\rfloor} k s_{k}^{(\nu)} s_{-k}^{(\nu)}+o(1)
$$

where $\Lambda_{\nu}$ is a constant to be determined.

Since $\log f^{(\nu)}(\theta)$ is a real odd function, its Fourier coefficients are imaginary and such that $s_{-k}^{(\nu)}=-s_{k}^{(\nu)}$. Therefore $s_{0}^{(\nu)}=0$ and the term linear in $N$ in the expression above cancels. Hence

$$
\log D_{N}\left[f^{(\nu)}\right]=\sum_{k=1}^{\left\lfloor N \Lambda_{\nu}\right\rfloor} k\left|s_{k}^{(\nu)}\right|^{2}+o(1) .
$$

One easily sees that the conjecture predicts a sublogarithmic growth of $\log D_{N}\left[f^{(\nu)}\right]$ with the dimension $N$. In fact, if we consider the asymptotic behaviour 20 of $s_{k}^{(\nu)}$ we have for $0<\nu<1 / 2$

$$
\log D_{N}\left[f^{(\nu)}\right]=\frac{\beta^{2}}{1-2 \nu}(\log N)^{1-2 \nu}+o(1)
$$

while for $\nu=1 / 2$

$$
\log D_{N}\left[f^{(1 / 2)}\right]=\beta^{2} \log \log N+o(1) .
$$

We can confront our conjecture (22) with the determinant evaluated numerically for large values of $N$, up to $10^{5}$. The results are summarized in Fig. 1. We have considered four values of $\nu(\nu=0,0.05,0.25$ and 0.50$)$ and $\beta=1 / \pi$. 


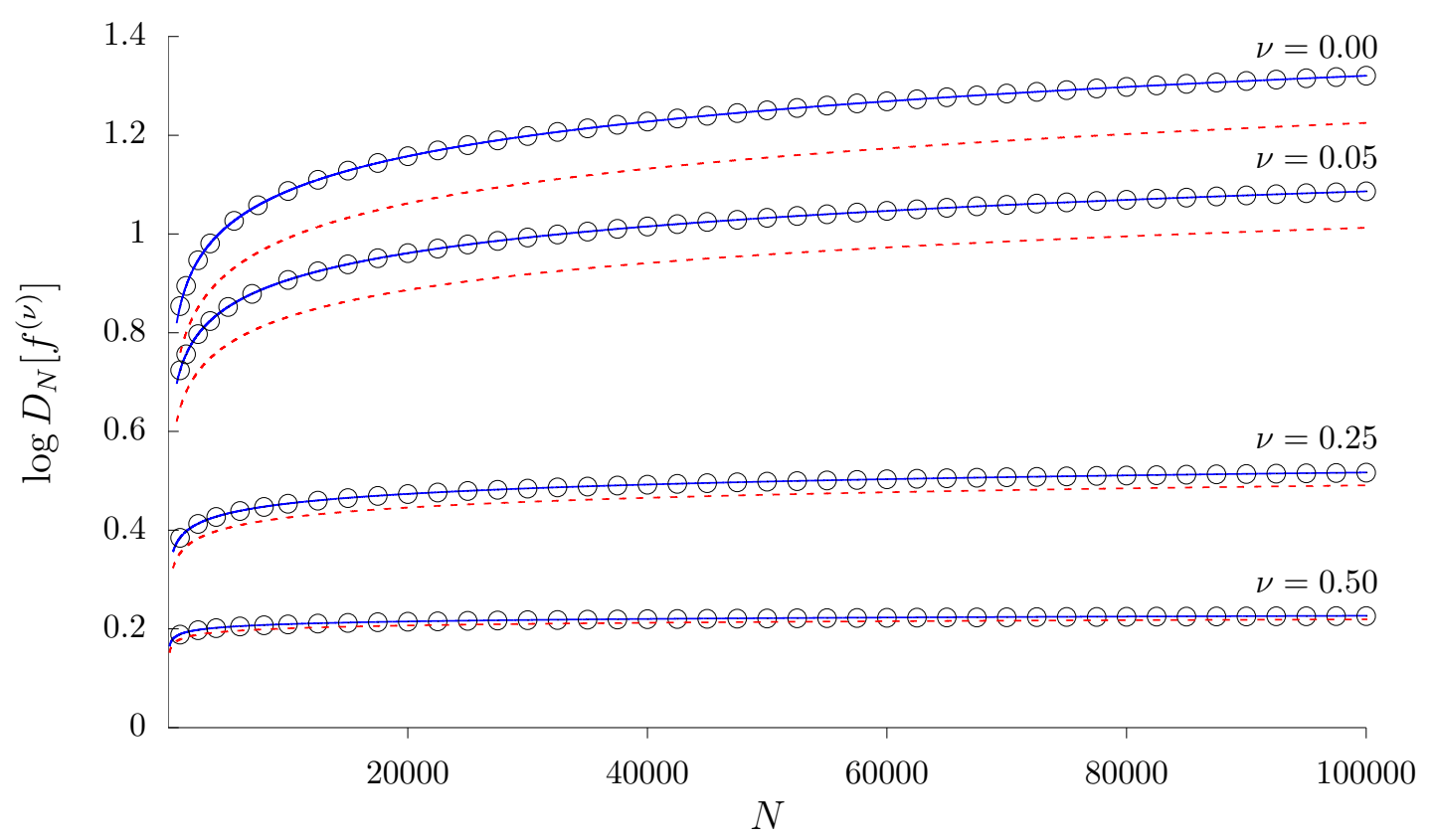

FIG. 1: Logarithm of the Toeplitz determinant with symbol $f^{(\nu)}$, defined in 19 , plotted against the dimension $N$. The dots correspond to the numerical values obtained using the supercomputer Memento for different exponents $\nu$ and $\beta=1 / \pi$. When $0 \leq \nu \leq 1 / 2, f^{(\nu)}$ does not satisfy the well-behavedness condition 11] of the Strong Szegő Theorem. The solid lines represent the conjecture proposed in 22 for the asymptotic behaviour of $\log D_{N}\left[f^{(\nu)}\right], \sum_{k=1}^{\left\lfloor N \Lambda_{\nu}\right\rfloor} k\left|s_{k}^{(\nu)}\right|^{2}$, with $\Lambda_{\nu}$ as given in Table I] For $\nu=0$ the symbol is discontinuous and we can apply the Fisher-Hartwig conjecture. In this case $\Lambda_{0}$ can be directly calculated using 18. The dashed lines correspond to the sum $\sum_{k=1}^{N} k\left|s_{k}^{(\nu)}\right|^{2}$, that is considering $\Lambda_{\nu}=1$.

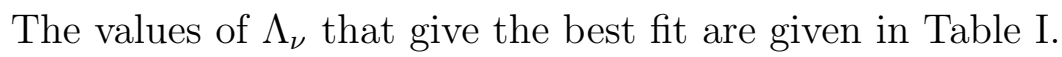

\begin{tabular}{ccccc}
\hline$\nu$ & 0.00 & 0.05 & 0.25 & 0.50 \\
$\Lambda_{\nu}$ & 2.566 & 2.599 & 2.659 & 2.660 \\
\hline
\end{tabular}

TABLE I: Values of $\Lambda_{\nu}$ that give the best fit of the curve $\sum_{k=1}^{\left\lfloor N \Lambda_{\nu}\right\rfloor} k\left|s_{k}^{(\nu)}\right|^{2}$ to the numerical points of Fig. 1 for the different values of $\nu$ and $\beta=1 / \pi$. The Fourier coefficients $s_{k}^{(\nu)}$ have been computed numerically. The value of $\Lambda_{\nu}$ for the case $\nu=0.00$ has been calculated using the expression $(18)$.

The accuracy of the fit is remarkable keeping in mind that it has been obtained by adjusting just one free parameter. For us this is a very strong indication that our conjecture is correct. 
In the following section we will apply this result to compute the entanglement entropy for a Kitaev chain with long range interaction.

\section{APPLICATION TO THE ENTANGLEMENT ENTROPY}

Here we will apply the results of the previous sections to study the asymptotic behaviour of the Rényi entanglement entropy of the following fermionic chain,

$H_{\log }=\sum_{m=1}^{M}\left(h a_{m}^{\dagger} a_{m}+a_{m}^{\dagger} a_{m+1}+a_{m+1}^{\dagger} a_{m}\right)+\sum_{m=1}^{M} \sum_{0<|l| \leq M / 2} \frac{1}{l(\log (A|l|))^{\nu}}\left(a_{m}^{\dagger} a_{m+l}^{\dagger}-a_{m} a_{m+l}\right)$,

where the pairing couplings decay logarithmically with an exponent $0 \leq \nu \leq 1 / 2$ and $A>1$. We shall consider $h \neq \pm 2$. Note that the value $\nu=0$ corresponds to the Long-Range Kitaev chain introduced in Section II.

In order to derive the asymptotic behaviour of the entanglement entropy for this system we shall need two technical results on block Toeplitz determinant that we borrow from different authors. For the purpose of keeping the paper readable yet self contained, we present these theorems, together with the necessary definitions, in the Appendix.

According to the general discussion of Section II, in the limit $M \rightarrow \infty$, the dispersion relation of $H_{\log }$ is

$$
\omega_{\log }(\theta)=\sqrt{F(\theta)^{2}+\left|G_{\nu}(\theta)\right|^{2}}
$$

where

$$
F(\theta)=h+2 \cos (\theta)
$$

and

$$
G_{\nu}(\theta)=\sum_{l=1}^{\infty} \frac{1}{l(\log (A|l|))^{\nu}}\left(\mathrm{e}^{\mathrm{i} \theta l}-\mathrm{e}^{-\mathrm{i} \theta l}\right) .
$$

Notice that the Fourier coefficients of $G_{\nu}, 1 /\left(l(\log A|l|)^{\nu}\right)$, are similar to the leading term in the expansion 20 for the Fourier coefficients $s_{k}^{(\nu)}$ of the function $\log f^{(\nu)}$ introduced in (19). Hence the behaviour of $G_{\nu}(\theta)$ in a neighbourhood of $\theta=0$ is equal to that of $\log f^{(\nu)}(\theta)$. We have seen that the divergence in the derivative of $\log f^{(\nu)}(\theta)$ at $\theta=0$ violates the well-behaviour condition (11) of the Strong Szegő Theorem when $0<\nu \leq 1 / 2$. This divergence gives rise to a sublogarithmic growth of the Toeplitz determinant with symbol $f^{(\nu)}$. Since $G_{\nu}(\theta)$ displays the same behaviour at $\theta=0$, we expect that this 
affects the asymptotics of the ground state entanglement entropy. We will show that this is indeed the case but we warn the reader that the derivation, that we outline in the rest of the section, is by no means immediate.

Following Ref. [41] we will compute the Rényi entanglement entropy for a subsystem $X$ of this chain using

$$
S_{\alpha, X}=\frac{1}{4 \pi \mathrm{i}} \lim _{\varepsilon \rightarrow 1^{+}} \oint_{\mathcal{C}} f_{\alpha}(\lambda / \varepsilon) \frac{\mathrm{d}}{\mathrm{d} \lambda} \log D_{X}(\lambda) \mathrm{d} \lambda,
$$

where $\mathcal{C}$ is the integration contour represented in Fig. 2 and $D_{X}(\lambda)=\operatorname{det}\left(\lambda I-V_{X}\right)$ with $V_{X}$ the restriction of the ground state correlation matrix to $X$,

$$
\left(V_{X}\right)_{n m}=2\left\langle\left(\begin{array}{c}
a_{n} \\
a_{n}^{\dagger}
\end{array}\right)\left(a_{m}^{\dagger}, a_{m}\right)\right\rangle-\delta_{n m} I, \quad n, m=1, \ldots,|X| .
$$

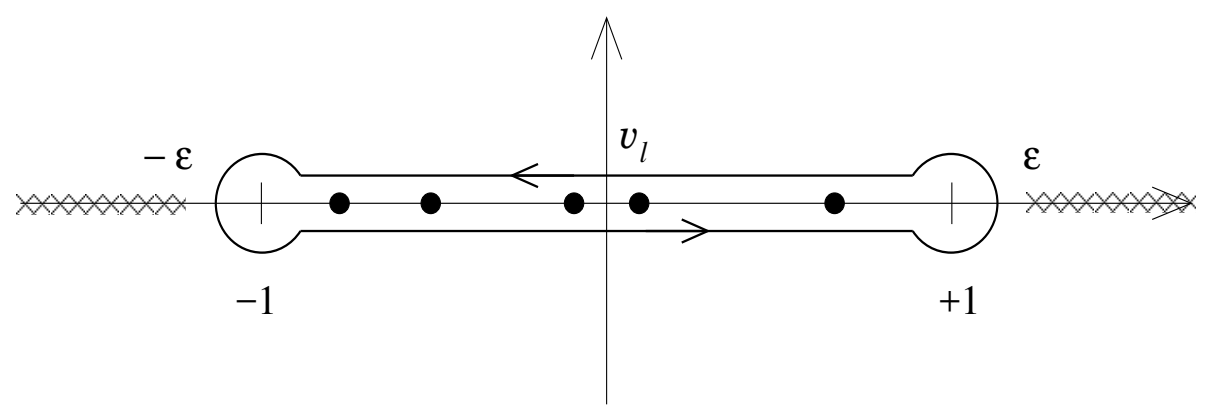

FIG. 2: Contour of integration, cuts and poles for the computation of $S_{\alpha, X}$ in 24 . The contour surrounds the eigenvalues $v_{l}$ of $V_{X}$, all of them lying on the real interval $[-1,1]$. The branch cuts for the function $f_{\alpha}$ extend to $\pm \infty$.

If the subsystem is a single interval of length $|X|$ then, in the limit $M \rightarrow \infty, V_{X}$ is a block Toeplitz matrix with entries

$$
\left(V_{X}\right)_{n m}=\frac{1}{2 \pi} \int_{-\pi}^{\pi} \mathcal{G}(\theta) \mathrm{e}^{\mathrm{i} \theta(n-m)} \mathrm{d} \theta, \quad n, m=1, \ldots,|X|,
$$

and symbol $\mathcal{G}(\theta)$, the $2 \times 2$ matrix

$$
\mathcal{G}(\theta)=\frac{1}{\omega_{\log }(\theta)}\left(\begin{array}{cc}
F(\theta) & G_{\nu}(\theta) \\
-G_{\nu}(\theta) & -F(\theta)
\end{array}\right)
$$

Our goal is to derive the leading term in the asymptotic expansion of the block Toeplitz determinant $D_{X}(\lambda)$ with symbol $\mathcal{G}_{\lambda}=\lambda I-\mathcal{G}$. The techniques developed in the previous 
section are valid for Toeplitz matrices, that is, when the symbol is a scalar function. Therefore, the first step in order to analyse the asymptotic behaviour of $D_{X}(\lambda)$ is to express it in terms of Toeplitz determinants.

For this purpose, let us perform the following change of basis,

$$
\mathcal{G}_{\lambda}(\theta)=\frac{1}{2}\left(\begin{array}{cc}
1 & 1 \\
-1 & 1
\end{array}\right) \mathcal{G}_{\lambda}^{\prime}(\theta)\left(\begin{array}{cc}
1 & -1 \\
1 & 1
\end{array}\right)
$$

where

$$
\mathcal{G}_{\lambda}^{\prime}(\theta)=\left(\begin{array}{cc}
\lambda & \mathrm{e}^{\mathrm{i} \xi(\theta)} \\
\mathrm{e}^{-\mathrm{i} \xi(\theta)} & \lambda
\end{array}\right)
$$

and

$$
\cos \xi(\theta)=\frac{F(\theta)}{\sqrt{F(\theta)^{2}+\left|G_{\nu}(\theta)\right|^{2}}}, \quad \sin \xi(\theta)=\frac{\mathrm{i} G_{\nu}(\theta)}{\sqrt{F(\theta)^{2}+\left|G_{\nu}(\theta)\right|^{2}}} .
$$

The determinants of the block Toeplitz matrices with $\mathcal{G}_{\lambda}$ and $\mathcal{G}_{\lambda}^{\prime}$ are equal and, therefore,

$$
\log D_{X}(\lambda)=\log D_{X}\left[\mathcal{G}_{\lambda}^{\prime}\right]
$$

In the following, it will be convenient to work with the symbol $\mathcal{G}_{\lambda}^{\prime}$ instead of $\mathcal{G}_{\lambda}$.

We introduce now the symbol

$$
\tilde{\mathcal{G}}_{\lambda}(\theta)=\left(\begin{array}{cc}
\lambda & \mathrm{e}^{\mathrm{i}|\xi(\theta)|} \\
\mathrm{e}^{-\mathrm{i}|\xi(\theta)|} & \lambda
\end{array}\right) .
$$

The behaviour of $|\xi(\theta)|$ in the neighbourhood of $\theta=0$ is similar to that of $\left|\log g_{\nu}(\theta)\right|$. If we calculate the leading term of the Fourier coefficients $\tilde{s}_{k}^{(\nu)}$ of $\left|\log g_{\nu}(\theta)\right|$ we find

$$
\tilde{s}_{k}^{(\nu)}=O\left(\frac{1}{k(\log |k|)^{\nu+1}}\right) .
$$

This means that

$$
\sum_{k=1}^{\infty} k \tilde{s}_{k}^{(\nu)} \tilde{s}_{-k}^{(\nu)}<\infty
$$

for any $\nu>0$ and it is well-behaved in the sense of (11) hence the Strong Szegö Theorem 12 is satisfied. Therefore, one may conclude that the symbol $\tilde{\mathcal{G}}_{\lambda}$ is also well-behaved and verifies the Widom theorem for block Toeplitz determinants A2, see the Appendix.

Since $\tilde{\mathcal{G}}_{\lambda}$ is well-behaved, we can apply the Basor localisation theorem A4 to the product of symbols $\mathcal{G}_{\lambda}^{\prime} \tilde{\mathcal{G}}_{\lambda}^{-1}$. Then we have

$$
\lim _{|X| \rightarrow \infty} \frac{D_{X}\left[\mathcal{G}_{\lambda}^{\prime} \tilde{\mathcal{G}}_{\lambda}^{-1}\right]}{D_{X}\left[\mathcal{G}_{\lambda}^{\prime}\right] D_{X}\left[\tilde{\mathcal{G}}_{\lambda}^{-1}\right]}<\infty
$$


This implies that

$$
\log D_{X}(\lambda)=\log D_{X}\left[\mathcal{G}_{\lambda}^{\prime}\right]=\log D_{X}\left[\mathcal{G}_{\lambda}^{\prime} \tilde{\mathcal{G}}_{\lambda}^{-1}\right]-\log D_{X}\left[\tilde{\mathcal{G}}_{\lambda}^{-1}\right]+O(1)
$$

Now, we can make use of Widom theorem A2 to derive the asymptotic behaviour of $\log D_{X}\left[\tilde{\mathcal{G}}_{\lambda}^{-1}\right]$. According to it,

$$
\log D_{X}\left[\tilde{\mathcal{G}}_{\lambda}^{-1}\right]=-\log \left(\lambda^{2}-1\right)|X|+O(1)
$$

because $\operatorname{det} \tilde{\mathcal{G}}_{\lambda}^{-1}(\theta)=\left(\lambda^{2}-1\right)^{-1}$. Therefore,

$$
\log D_{X}(\lambda)=\log D_{X}\left[\mathcal{G}_{\lambda}^{\prime} \tilde{\mathcal{G}}_{\lambda}^{-1}\right]+\log \left(\lambda^{2}-1\right)|X|+O(1)
$$

Note that, by the definition of $\tilde{\mathcal{G}_{\lambda}}$, the product $\mathcal{G}_{\lambda}^{\prime} \tilde{\mathcal{G}}_{\lambda}^{-1}$ is

$$
\mathcal{G}_{\lambda}^{\prime}(\theta) \tilde{\mathcal{G}}_{\lambda}(\theta)^{-1}=I \text {, for }-\pi \leq \theta<0
$$

and

$$
\mathcal{G}_{\lambda}^{\prime}(\theta) \tilde{\mathcal{G}}_{\lambda}(\theta)^{-1}=\frac{1}{\lambda^{2}-1}\left(\begin{array}{cc}
\lambda^{2}-\mathrm{e}^{2 \mathrm{i} \xi(\theta)} & 2 \mathrm{i} \lambda \sin \xi(\theta) \\
-2 \mathrm{i} \lambda \sin \xi(\theta) & \lambda^{2}-\mathrm{e}^{-2 \mathrm{i} \xi(\theta)}
\end{array}\right), \text { for } 0 \leq \theta<\pi .
$$

Then the unitary matrix

$$
U(\theta)=\frac{1}{\sqrt{2}}\left(\begin{array}{cc}
U_{+}(\theta) & U_{-}(\theta) \\
U_{-}(\theta) & U_{+}(\theta)
\end{array}\right)
$$

with

$$
U_{ \pm}(\theta)=\left(1 \pm \sqrt{1-\lambda^{2} \sec |\xi(\theta)|}\right)^{1 / 2}
$$

diagonalizes the symbol $\mathcal{G}_{\lambda}^{\prime} \tilde{\mathcal{G}}_{\lambda}^{-1}$,

$$
U(\theta) \mathcal{G}_{\lambda}^{\prime} \tilde{\mathcal{G}}_{\lambda}^{-1} U(\theta)^{-1}=\left(\begin{array}{cc}
\mu_{\lambda}^{-}(\theta) & 0 \\
0 & \mu_{\lambda}^{+}(\theta)
\end{array}\right)
$$

where the eigenvalues are

$$
\mu_{\lambda}^{ \pm}(\theta)=1, \text { for }-\pi \leq \theta<0,
$$

and

$$
\mu_{\lambda}^{ \pm}(\theta)=\left(\frac{\sqrt{\lambda^{2}-\cos ^{2} \xi(\theta)} \pm \sin \xi(\theta)}{\sqrt{\lambda^{2}-1}}\right)^{2}, \text { for } 0 \leq \theta<\pi
$$


Since $U(\theta)$ only depends on $|\xi(\theta)|$ we may conclude that it is well-behaved by the same reasons as those given for the symbol $\tilde{\mathcal{G}}_{\lambda}$. Then we can apply again the localisation theorem $\mathrm{A} 4$ to the product $U \mathcal{G}_{\lambda}^{\prime} \tilde{\mathcal{G}}_{\lambda}^{-1} U^{-1}$, and

$$
\log D_{X}\left[\mathcal{G}_{\lambda}^{\prime} \tilde{\mathcal{G}}_{\lambda}^{-1}\right]=\log D_{X}\left[U \mathcal{G}_{\lambda}^{\prime} \tilde{\mathcal{G}}_{\lambda}^{-1} U^{-1}\right]-\log D_{X}[U]-\log D_{X}\left[U^{-1}\right]+O(1)
$$

Due to the well-behaviour of $U$, the Widom theorem $\mathrm{A} 2$ gives the asymptotic limit of $\log D_{X}[U]$ and $\log D_{X}\left[U^{-1}\right]$. In fact, since $\operatorname{det} U(\theta)=1$, the linear, dominant term of their expansion in $|X|$ vanishes and the rest of the terms tend to a finite value when $|X| \rightarrow \infty$. This leads us to conclude that

$$
\log D_{X}\left[\mathcal{G}_{\lambda}^{\prime} \tilde{\mathcal{G}}_{\lambda}^{-1}\right]=\log D_{X}\left[U \mathcal{G}_{\lambda}^{\prime} \tilde{\mathcal{G}}_{\lambda}^{-1} U^{-1}\right]+O(1)
$$

Notice that the product $U \mathcal{G}_{\lambda}^{\prime} \tilde{\mathcal{G}}_{\lambda}^{-1} U^{-1}$ is diagonal and, therefore, the corresponding block Toeplitz matrix can be expressed, after a global change of basis, as the direct sum of two Toeplitz matrices with symbol the eigenvalues $\mu_{\lambda}^{+}(\theta)$ and $\mu_{\lambda}^{-}(\theta)$. This fact implies that

$$
\log D_{X}\left[U \mathcal{G}_{\lambda}^{\prime} \tilde{\mathcal{G}}_{\lambda}^{-1} U^{-1}\right]=\log D_{X}\left[\mu_{\lambda}^{+}\right]+\log D_{X}\left[\mu_{\lambda}^{-}\right]
$$

that allows to apply the conjecture for Toeplitz determinants with anomalous symbol that we have proposed in Section III. According to it the Toeplitz determinants with symbol $\mu_{\lambda}^{ \pm}$behave as

$$
\log D_{X}\left[\mu_{\lambda}^{ \pm}\right]=s_{0}^{ \pm}(\lambda)|X|+\sum_{k=1}^{|X|} k s_{k}^{ \pm}(\lambda) s_{-k}^{ \pm}(\lambda)+o(1)
$$

where

$$
s_{k}^{ \pm}(\lambda)=\frac{1}{2 \pi} \int_{-\pi}^{\pi} \log \mu_{\lambda}^{ \pm}(\theta) \mathrm{e}^{\mathrm{i} \theta k} \mathrm{~d} \theta
$$

are the Fourier coefficients of the logarithm of the eigenvalues $\mu_{\lambda}^{ \pm}(\theta)$.

Since the zero modes satisfy $s_{0}^{+}(\lambda)=-s_{0}^{-}(\lambda)$ the linear terms of the expansion 30 cancel in 29) and, therefore,

$$
\log D_{X}\left[U \mathcal{G}_{\lambda}^{\prime} \tilde{\mathcal{G}}_{\lambda}^{-1} U^{-1}\right]=\sum_{k=1}^{|X|} k\left[s_{k}^{+}(\lambda) s_{-k}^{+}(\lambda)+s_{k}^{-}(\lambda) s_{-k}^{-}(\lambda)\right]+O(1) .
$$

Collecting (27), 28) and (31), we conclude that the asymptotic behaviour of $\log D_{X}(\lambda)$ is given by

$$
\log D_{X}(\lambda)=\log \left(\lambda^{2}-1\right)|X|+\sum_{k=1}^{|X|} k\left[s_{k}^{+}(\lambda) s_{-k}^{+}(\lambda)+s_{k}^{-}(\lambda) s_{-k}^{-}(\lambda)\right]+O(1) .
$$


Now, if we insert the linear term of this expansion in the contour integral (24) it vanishes [? ] and, therefore, the leading contribution to the entanglement entropy is given by the sum.

Hence to obtain the growth rate of the latter we need to study the asymptotic behaviour of the Fourier coefficients, which are given by

$$
s_{k}^{ \pm}(\lambda)=\frac{1}{\pi} \int_{0}^{\pi} \log \frac{\sqrt{\lambda^{2}-\cos ^{2} \xi(\theta)} \pm \sin \xi(\theta)}{\sqrt{\lambda^{2}-1}} \mathrm{e}^{\mathrm{i} k \theta} \mathrm{d} \theta
$$

where $\cos \xi(\theta)$ and $\sin \xi(\theta)$ are as given in 26 and we have used that $\mu_{\lambda}^{ \pm}(\theta)=1$ for $-\pi \leq \theta<0$.

Standard estimation techniques, like the change of variable to $k \theta$ and the rotation of the integration domain to the imaginary axis, lead to the following asymptotic behaviour

$$
s_{k}^{ \pm}(\lambda)=\frac{\mathrm{i}}{k \pi} \log \left(1 \mp \frac{\pi /(h+2)}{(\log |A k|)^{\nu} \sqrt{\lambda^{2}-1}}\right)+O\left(\frac{1}{(\log |k|)^{\nu+1}}\right) .
$$

Now we are equipped with all the ingredients to derive the asymptotic behaviour of the Rényi entanglement entropy.

First notice that, as it was established before, the linear term vanishes and the contour integral reduces to

$$
S_{\alpha, X}=\frac{1}{4 \pi \mathrm{i}} \lim _{\varepsilon \rightarrow 1^{+}} \oint_{\mathcal{C}} f_{\alpha}(\lambda / \varepsilon) \frac{\mathrm{d}}{\mathrm{d} \lambda} E_{X}(\lambda) \mathrm{d} \lambda
$$

where

$$
E_{X}(\lambda)=\sum_{k=1}^{|X|} k\left[s_{k}^{+}(\lambda) s_{-k}^{+}(\lambda)+s_{k}^{-}(\lambda) s_{-k}^{-}(\lambda)\right]+O(1)
$$

Performing an integration by parts, one obtains

$$
S_{\alpha, X}=-\frac{1}{4 \pi \mathrm{i}} \lim _{\varepsilon \rightarrow 1^{+}} \oint_{\mathcal{C}} \frac{\mathrm{d} f_{\alpha}(\lambda / \varepsilon)}{\mathrm{d} \lambda} E_{X}(\lambda) \mathrm{d} \lambda .
$$

Since $s_{k}^{ \pm}(\lambda)$ is analytic outside the real interval $[-1,1]$, we can evaluate the latter integral by deforming the integration contour to infinity, surrounding the poles and cuts of $\mathrm{d} f_{\alpha} / \mathrm{d} \lambda$ as it is represented in Fig. 3. Inserting in $E_{X}$ the asymptotic behaviour of $s_{k}$ given in 33 and replacing the sum $\sum_{k=1}^{|X|}$ by an integral $\int_{0}^{|X|} \mathrm{d} k$ we can, in principle, obtain the asymptotic behaviour for $S_{\alpha, X}$. 


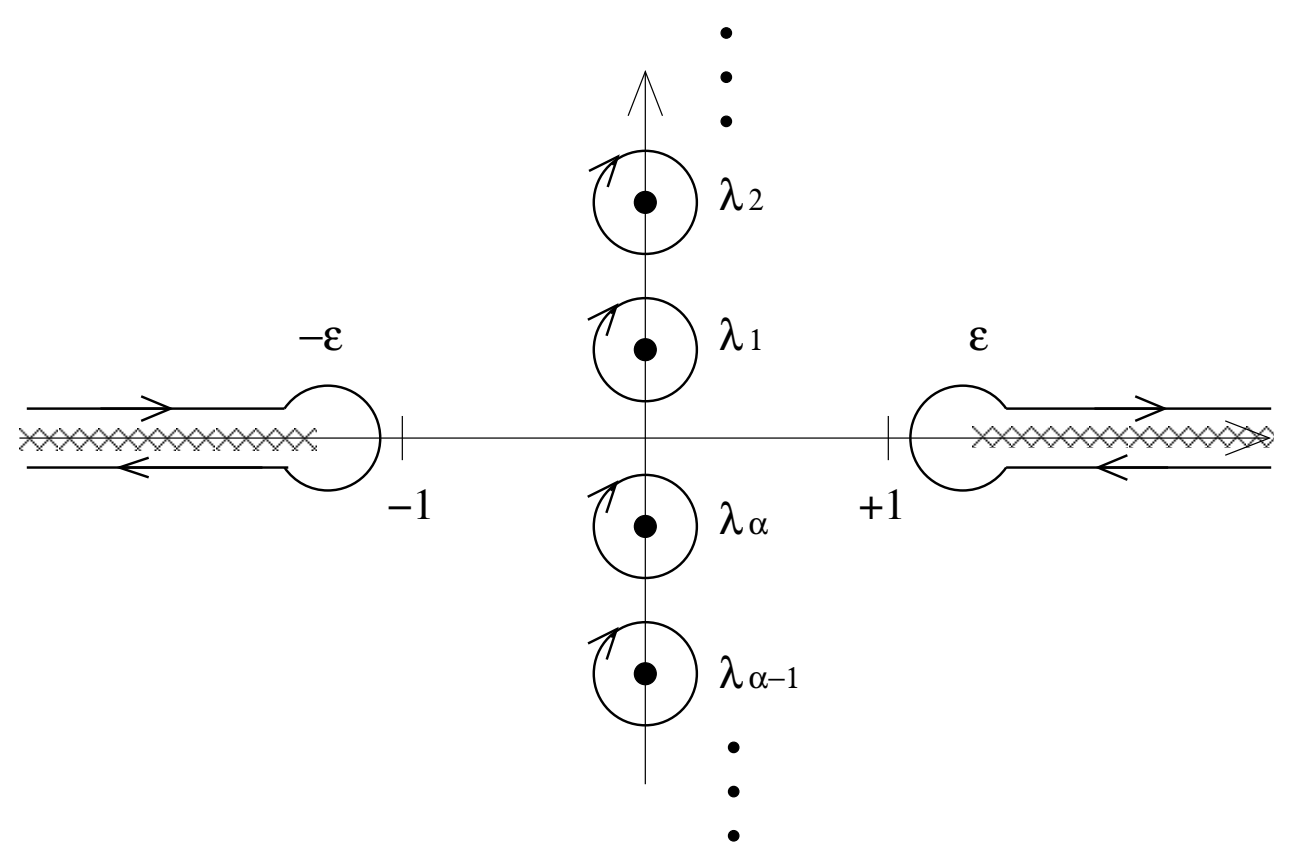

FIG. 3: Contours of integration for the computation of the Rényi entanglement entropy in 35. They surround clockwise the poles and branch cuts of the function $\mathrm{d} f_{\alpha}(\lambda / \varepsilon) / \mathrm{d} \lambda$. Poles are the points at the imaginary axis $\lambda_{l}$ given by $[36$ while branch cuts correspond to the intervals $(-\infty,-\varepsilon]$ and $[\varepsilon, \infty)$.

While the previous computation as it has been described, is perfectly feasible, we obtain rather cumbersome expressions that add little understanding to our study of the sublogarithmic law for the entanglement entropy. However, when $\alpha$ is an integer greater than 1 the problem simplifies notably and we are able to give very explicit formulae.

In this case the cuts in Fig. 3 disappear and the only singularities of $\mathrm{d} f_{\alpha}(\lambda) / \mathrm{d} \lambda$ are simple poles at the points of the imaginary axis

$$
\lambda_{l}=\mathrm{i} \tan \frac{\pi(2 l-1)}{2 \alpha}, \quad l=1, \ldots, \alpha, \quad l \neq \frac{\alpha+1}{2} .
$$

Therefore, $S_{\alpha, X}$ can be computed from the sum of the residues of the integrand in (35) at these points,

$$
S_{\alpha, X}=\frac{1}{2(1-\alpha)} \sum_{l=1}^{\alpha} E_{X}\left(\lambda_{l}\right)
$$

Using the asymptotic behaviour of $s_{k}^{ \pm}$given in 33 and restricting to first order we have

$$
k s_{k}^{ \pm}\left(\lambda_{l}\right) s_{-k}^{ \pm}\left(\lambda_{l}\right)=-\frac{c\left(\lambda_{l}\right)}{k(\log |A k|)^{2 \nu}}+O\left(\frac{1}{k(\log |k|)^{3 \nu}}\right)
$$

where

$$
c(\lambda)=\frac{1}{(h+2)^{2}\left(|\lambda|^{2}+1\right)}
$$


If we plug this into (37) and approximate the sum over $k$ by an integral, we obtain that the Rényi entanglement entropy for integer $\alpha>1$ grows with $|X|$ as

$$
S_{\alpha, X}=C_{\alpha}^{(\nu)}(\log |X|)^{1-2 \nu}+O\left((\log |X|)^{1-3 \nu}+1\right), \quad \text { for } \quad 0<\nu<1 / 2,
$$

where

$$
\begin{aligned}
C_{\alpha}^{(\nu)} & =\frac{1}{\alpha-1} \sum_{l=1}^{\alpha} \frac{c\left(\lambda_{l}\right)}{1-2 \nu} \\
& =\frac{\alpha}{2(\alpha-1)} \frac{1}{(h+2)^{2}(1-2 \nu)} .
\end{aligned}
$$

On the other hand,

$$
S_{\alpha, X}=C_{\alpha}^{(1 / 2)} \log \log |X|+O(1), \quad \text { for } \quad \nu=1 / 2
$$

with

$$
\begin{aligned}
C_{\alpha}^{(1 / 2)} & =\frac{1}{\alpha-1} \sum_{l=1}^{\alpha} c\left(\lambda_{l}\right) \\
& =\frac{\alpha}{2(\alpha-1)} \frac{1}{(h+2)^{2}} .
\end{aligned}
$$

In conclusion, we expect a sublogarithmic growth of the Rényi entanglement entropy in the ground state of the Hamiltonian (23) with logarithmic decaying pairings. To our knowledge, this kind of behaviour has not been reported in the literature. A striking feature of our result is that the next terms in the expansion $(38)$ are of the form $(\log |X|)^{1-m \nu}$ with $m=3,4, \ldots$. Therefore, all the terms for which $m<1 / \nu$ also diverge in the limit $|X| \rightarrow \infty$. This makes that very similar, divergent terms mix up and, therefore, it is difficult to isolate numerically the leading asymptotic behaviour. Hence, in order to test our predictions we must allow for subdominat terms.

In Fig. 4 we represent the Rényi entanglement entropy for $\alpha=2$ with $\nu=0.25$ (squares) and $\nu=0.5$ (circles), taking in both cases $h=1 / 2$ and $A=2$, as a function of the size of the subsystem $|X|$. The curves are obtained by supplementing the predicted coefficient for the leading asymptotic term of $(39)$ and 40$)$ with the next two subleading corrections. The values of the latter coefficients have been computed by fitting the curve to the numerical results in the interval between $|X|=100$ to $|X|=1000$.

The continuous line, associated to $\nu=0.25$ represents the function

$$
F_{0.25}(x)=\frac{8}{25}(\log x)^{\frac{1}{2}}+a(\log x)^{\frac{1}{4}}+b, \quad \text { with } \quad a=-0.400, b=0.811 .
$$


The dot-dashed line corresponds to $\nu=0.5$ and is given by

$$
F_{0.50}(x)=\frac{4}{25} \log \log x+c+d(\log x)^{-\frac{1}{2}}, \quad \text { with } \quad c=0.474, d=0.256
$$

Note that the curves are very close to the numerical results. This fact is specially noticeable if we consider that the fit for $a, b, c$ and $d$ has been performed in the window between 100 and 1000 and then we extend the curve up to $|X|=10000$. We believe that this extraordinary agreement is a strong support for the validity of our results.

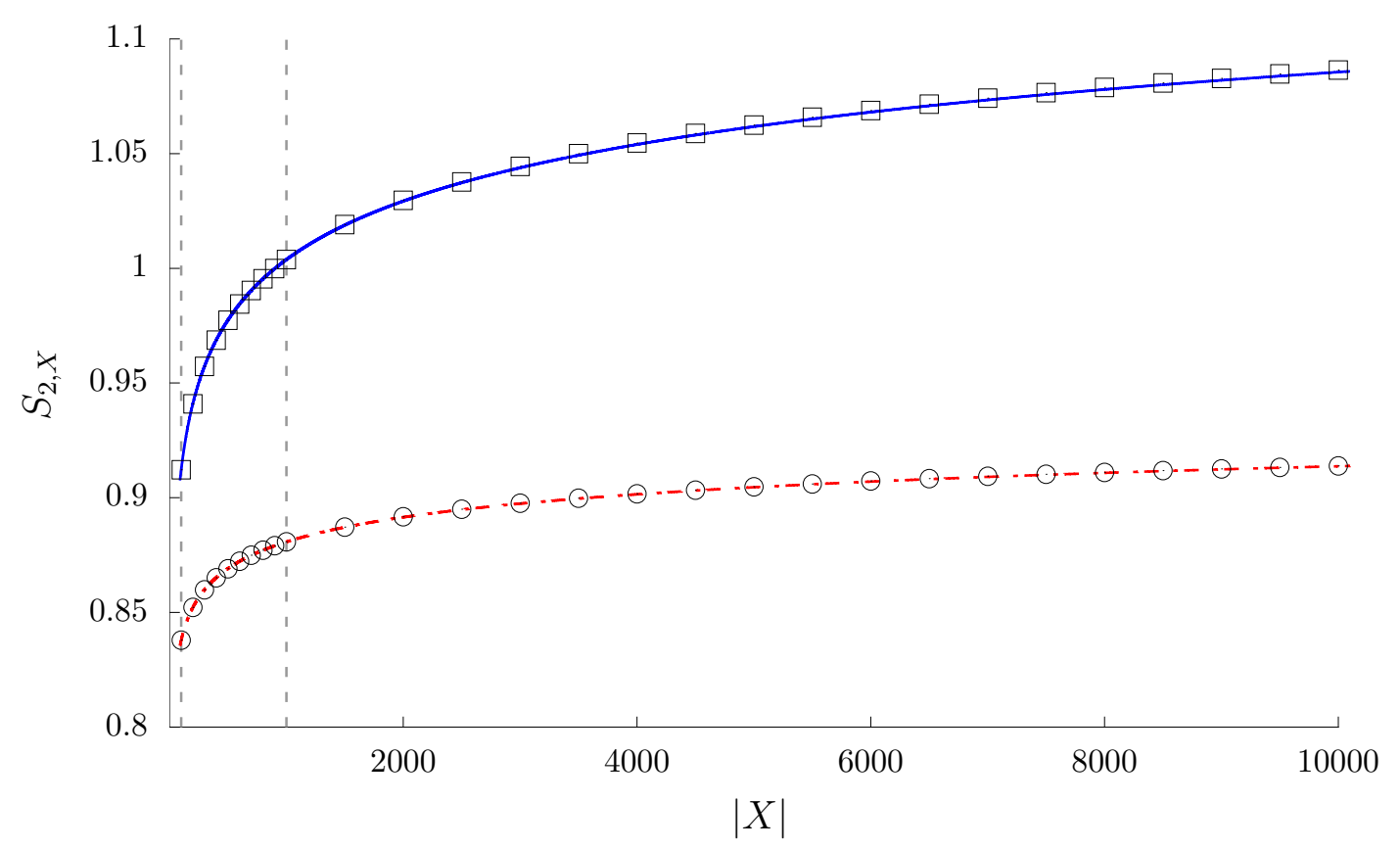

FIG. 4: The squares and circles represent the numerical value of the Rényi entanglement entropy for $\alpha=2$ with $\nu=0.25$ and $\nu=0.5$ respectively as a function of $|X|$. The continuous line contains our prediction for the leading term for $\nu=0.25$ supplemented with the two next subleading corrections $F_{0.25}(x)=\frac{8}{25}(\log x)^{\frac{1}{2}}-0.400(\log x)^{\frac{1}{4}}+0.811$. The dot-dashed line is the analogous for $\nu=0.5$, i. e. $F_{0.50}(x)=\frac{4}{25} \log \log x+0.474+0.256(\log x)^{-\frac{1}{2}}$.

\section{CONCLUSIONS}

In this paper we have investigated the possibility of unexplored behaviours for the Rényi entanglement entropy in fermionic chains, different from the linear and the logarithmic growth or the saturation to a constant value with the length of the interval. 
Our investigation is based on the relation between block Toeplitz determinants and the Rényi entanglement entropy in the ground state of quadratic, homogeneous fermionic chains. In particular, we have considered chains where the pairing couplings decay logarithmically with the distance. For these systems, the ground state entanglement entropy is related to the Toeplitz determinant generated by a family of continuous, non-zero symbols that do not lie within the domain of validity of the Strong Szegö theorem.

We have proposed a new asymptotic regime for these symbols that leads to the sublogarithmic growth of the logarithm of the Toeplitz determinant and, therefore, of the Rényi entanglement entropy. That is, the leading term of the ground state entanglement entropy in these fermionic chains is $(\log |X|)^{a}$ for some $0<a<1$ related to the dumping exponent in the pairing couplings, and $|X|$ the length of the interval in the chain. We have also found a case with a $\log \log |X|$ behaviour.

The numerical tests performed support the conjecture for this type of Toeplitz determinants.

It is remarkable that this asymptotic regime both for Toeplitz determinants and for the entanglement entropy has not been previously reported in the literature. In the logarithmic conformal field theories studied in [25], they found terms of the form $\log \log |X|$ but, contrary to our case, they are subleading, see also [26, 27]. The same occurs with the sublogarithmic terms detected numerically in 28] in long range quantum Ising chains. It would be interesting to understand the leading sublogarithmic terms that we have found here using field theory methods as well as to investigate if they appear in other models, and in particular, whether there is any direct relation between our results for $\nu=1 / 2$ and some logarithmic CFT.

Acknowledgments: Research partially supported by grants E21_17R, DGIIDDGA and FPA2015-65745-P, MINECO (Spain). FA was supported by FPI Grant No. C070/2014, DGIID-DGA/European Social Fund. ZZ was supported by the Janos Bolyai Scholarship, and the National Research Development and Innovation Office of Hungary under Project No. 2017-1.2.1-NKP-2017-00001, and by Grants No. K124152 and KH129601. The authors thankfully acknowledge the resources from the supercomputer Memento, technical expertise and assistance provided by BIFI (Universidad de Zaragoza). 


\section{Appendix A}

In this Appendix we will present several results on, so called, block Toeplitz determinants that are necessary for the proper understanding of the paper.

A block Toeplitz matrix with $d$-dimensional matrix valued symbol $J: S^{1} \rightarrow M_{d}(\mathbb{C})$ is a $(N \cdot d) \times(N \cdot d)$ matrix formed by $d$ dimensional blocks which are given by the Fourier coefficients of $J$, namely

$$
\left(T_{N}[J]\right)_{n m}=J_{n-m}=\frac{1}{2 \pi} \int_{-\pi}^{\pi} J(\theta) \mathrm{e}^{\mathrm{i} \theta(n-m)} \mathrm{d} \theta, \quad n, m=1, \ldots, N .
$$

We will be mainly interested in the determinant of this matrix that we will denote by $D_{N}[J]=\operatorname{det}\left(T_{N}[J]\right)$.

Gyires [57] found a generalisation of the Szegö theorem 10 for the determinant of block Toeplitz matrices that was later extended by Hirschman [58] and Widom [59] to a wider variety of symbols. According to this, the leading term in the asymptotic expansion of $\log D_{N}[J]$ should be also linear

$$
\log D_{N}[J]=\frac{N}{2 \pi} \int_{-\pi}^{\pi} \log \operatorname{det} J(\theta) \mathrm{d} \theta+o(N),
$$

provided $\operatorname{det} J(\theta) \neq 0$ and the argument of $\operatorname{det} J(\theta)$ is continuous and periodic for $\theta \in$ $[-\pi, \pi]$ (zero winding number).

Analogously to the scalar case, if in addition the symbol $J(\theta)$ is well-behaved in the sense that

$$
\sum_{k=-\infty}^{\infty}\left\|J_{k}\right\|+\sum_{k=-\infty}^{\infty}|k|\left\|J_{k}\right\|^{2}<\infty
$$

where $\|\cdot\|$ is the Hilbert-Schmidt norm of the $d \times d$ matrices, the Widom theorem [59 61] states that the next contribution in the expansion (A1) should be finite when $|X| \rightarrow \infty$,

$$
\log D_{N}[J]=\frac{N}{2 \pi} \int_{-\pi}^{\pi} \log \operatorname{det} J(\theta) \mathrm{d} \theta+E[J]+o(1)
$$

If we call $T[J]$ the semi-infinite matrix obtained from $T_{N}[J]$ when $N \rightarrow \infty$, the constant term $E[J]$ reads

$$
E[J]=\log \operatorname{det} T[J] T\left[J^{-1}\right] .
$$

The Widom theorem reduces to the Strong Szegö theorem (12) when the symbol is a scalar function, i.e. $d=1$. This result will be important for us in order to find the asymptotic behaviour of the entanglement entropy for the long range Kitaev chain (23). 
Another theorem that we will be needed troughout the paper is the localisation theorem found by E. Basor in [56]. It reads as follows:

If we consider two $d \times d$ symbols $J_{1}(\theta), J_{2}(\theta)$ such that their block Toeplitz matrices $T_{X}\left[J_{1}\right]$ and $T_{X}\left[J_{2}\right]$ are invertible for $|X|$ large enough and the semi-infinite matrices $T\left[J_{1} J_{2}\right]-T\left[J_{1}\right] T\left[J_{2}\right], T\left[J_{2} J_{1}\right]-T\left[J_{2}\right] T\left[J_{1}\right]$ are trace-class then

$$
\lim _{|X| \rightarrow \infty} \frac{D_{X}\left[J_{1} J_{2}\right]}{D_{X}\left[J_{1}\right] D_{X}\left[J_{2}\right]}<\infty
$$

The operator $T\left[J_{1} J_{2}\right]-T\left[J_{1}\right] T\left[J_{2}\right]$ is trace-class if there exists a smooth partition of the unit $\left\{f_{1}(\theta), f_{2}(\theta)\right\}$ such that the derivatives of $J_{1} f_{1}$ and $J_{2} f_{2}$ are Hölder continuous with an exponent greater than $1 / 2$. Or, in more informally words, the operator $T\left[J_{1} J_{2}\right]-T\left[J_{1}\right] T\left[J_{2}\right]$ is trace-class provided $J_{1}$ and $J_{2}$ are not bad (non smooth) at the same point.

The localisation theorem implies that if the symbol $J(\theta)$ has several discontinuities at $\theta_{1}, \ldots, \theta_{R}$, the divergent contribution in the large $|X|$ limit of each one to $D_{X}[J]$ is independent from the rest and they can be studied separately.

Although it is not really necessary in this paper, for the sake of completeness we would like to mention one more result found in [12] that allows to compute the logarithmic divergence of block Toeplitz determinants with symbols that have jump discontinuities.

This is a generalization of the scalar case mentioned in Section III and can be stated as follows:

Let $J$ be a piecewise smooth $d \times d$ matrix valued symbol such that $\operatorname{det} J(\theta) \neq 0$ and has zero winding number. If $J$ has jump discontinuities at $\theta_{1}, \ldots, \theta_{R}$, where $J_{r}^{ \pm}$are the lateral limits of $J(\theta)$ at $\theta_{r}$, the determinant $D_{X}[J]$ of its block Toeplitz matrix is

$$
\log D_{X}[J]=\frac{|X|}{2 \pi} \int_{-\pi}^{\pi} \log \operatorname{det} J(\theta) \mathrm{d} \theta+\frac{\log |X|}{4 \pi^{2}} \sum_{r=1}^{R} \operatorname{Tr}\left(\log \left(J_{r}^{-}\left(J_{r}^{+}\right)^{-1}\right)^{2}\right)+O(1) .
$$

[1] J. D. Bekenstein, Black Holes and Entropy, Phys. Rev. D 7, 2333 (1973)

[2] S. Hawking, Particle creation by black holes, Comm. Math. Phys. 43, 3, 199 (1975)

[3] L. Bombelli, R. K. Koul, J. Lee, R. D. Sorkin, Quantum source of entropy for black holes, Phys. Rev. D 34, 373 (1986)

[4] M. Srednicki, Entropy and Area, Phys. Rev. Lett. 71, 666 (1993), arXiv:hep-th/9303048 
[5] M. B. Hastings, An area law for one-dimensional quantum systems, J. Stat. Mech. (2007) P08024, arXiv:0705.2024 [quant-ph]

[6] C. Holzhey, F. Larsen, F. Wilczek, Geometric and renormalized entropy in conformal field theory, Nucl. Phys. B 424, 443-467 (1994), hep-th/9403108

[7] P. Calabrese, J. Cardy, Entanglement Entropy and Quantum Field Theory, J. Stat. Mech. (2004) P06002, arXiv:hep-th/0405152

[8] M. M. Wolf, Violation of entropic area law for fermions, Phys. Rev. Lett. 96, 010404 (2006), arXiv: quant-ph/0503219

[9] F. Ares, J. G. Esteve, F. Falceto, E. Sánchez-Burillo, Excited state entanglement in homogeneous fermionic chains, J. Phys. A: Math. Theor. 47 (2014) 245301, arXiv:1401.5922 [quan-phys]

[10] D. Vodola, L. Lepori, E. Ercolessi, A. V. Gorshkov, G. Pupillo, Kitaev chains with longrange pairing, Phys. Rev. Lett. 113, 156402 (2014), arXiv:1405.5440 [cond-mat.str-el]

[11] F. Ares, J. G. Esteve, F. Falceto, A. R. de Queiroz, Entanglement in fermionic chains with finite range coupling and broken symmetries, Phys. Rev. A 92, 042334 (2015),

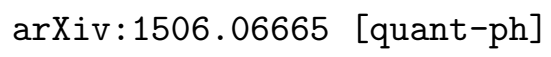

[12] F. Ares, J. G. Esteve, F. Falceto, A. R. de Queiroz, Entanglement entropy in the Long-Range Kitaev chain, Phys. Rev. A 97, 062301 (2018), arXiv:1801.07043 [quant-ph]

[13] G. Vitagliano, A. Riera, J. I. Latorre, Violations of the area-law scaling for the entanglement entropy in spin 1/2 chains, New J. Phys.,12(11):113049, 2010. arXiv:1003.1292v2 [quant-ph]

[14] G. Ramirez, J. Rodriguez-Laguna, G. Sierra, From conformal to volume law for the entanglement entropy in exponentially deformed critical spin 1/2 chains, J. Stat. Mech. 2014 (10): P10004. arXiv:1407.3456 [quant-ph]

[15] J. Dubail, J. M. Stephan, J. Viti, P. Calabrese, Conformal field theory for inhomogeneous one-dimensional quantum systems: the example of non-interacting fermi gases, SciPost Phys., 2:002, 2017 arXiv:1606.04401 [cond-mat.str-el]

[16] V. Alba, S. N. Santalla, P. Ruggiero, J. Rodriguez-Laguna, P. Calabrese, G. Sierra, Unusual area-law violation in random inhomogeneous systems, J. Stat. Mech. 2019:023105 arXiv:1807.11222 [quant-ph]

[17] G. Refael, J. E. Moore, Entanglement entropy of random critical points in one dimension, 
Phys. Rev. Lett. 93, 260602, (2004). arXiv:046737 [cond-mat]

[18] N. Laflorencie, Scaling of entanglement entropy in the random singlet phase, Phys. Rev. B 72, 140408(R), (2005). arXiv:0504446 [cond-mat]

[19] R. Movassagh, P. W. Shor, Power law violation of the area law in quantum spin chains, PNAS (2016): 201605716, arXiv:1408.1657 [quant-ph]

[20] O. Salberger, V. E. Korepin, Fredkin Spin Chain, arXiv:1605.03842 [quant-ph]

[21] O. Salberger, T. Udagawa, Z. Zhang, H. Katsura, I. Klich, V. E. Korepin, Deformed Fredkin spin chain with extensive entanglement, J. Stat. Mech. (2017) 063103, arXiv:1611.04983 [cond-mat.stat-mech]

[22] M. Fannes, B. Haegeman, M. Mosonyi, Entropy growth of shift-invariant states on a quantum spin chain, J. Math. Phys. 44, 6005-6019 (2003), arXiv:math-ph/0306055

[23] S. Farkas, Z. Zimborás, On the sharpness of the zero-entropy-density conjecture, J. Math. Phys. 46, 123301 (2005), arXiv:math-ph/0507022

[24] G. Gori, S. Paganelli, A. Sharma, P. Sodano, A. Trombettoni, Bell-paired states inducing volume law for entanglement entropy in fermionic lattices, Phys. Rev. B 91, 245138 (2015), arXiv:1405.3616 [cond-mat.stat-mech]

[25] D. Bianchini, O. Castro-Alvaredo, B. Doyon, E. Levi, F. Ravanini, Entanglement entropy of non-unitary conformal field theory, J. Phys. A: Math. Theor. 48 04FT01 (2015), arXiv:1405.2804 [hep-th]

[26] R. Couvreur, J. L. Jacobsen, H. Saleur, Entanglement in non-unitary quantum critical spin chains, Phys. Rev. Lett. 119, 040601, 2017, arXiv:1611.08506 [cond-mat.stat-mech]

[27] J. C. Xavier, F. C. Alcaraz, G. Sierra, Equipartition of the entanglement entropy, Phys. Rev. B 98, 041106(R), 2018, arXiv:1804.06357 [cond-mat.stat-mech]

[28] T. Koffel, M. Lewenstein, L. Tagliacozzo, Entanglement Entropy for the Long-Range Ising Chain in a Transverse Field, Phys. Rev. Lett. 109, 267203 (2012), arXiv:1207.3957 [cond-mat.str-el]

[29] D. Vodola, L. Lepori, E. Ercolessi, and G. Pupillo, Long-Range Ising and Kitaev Models: Phases, Correlations and Edge Modes, New J. Phys. 18, 015001 (2016), arXiv:1508.00820 [cond-mat.str-el]

[30] S. Hernández-Santana, C. Gogolin, J. I. Cirac, A. Acín, Correlation decay in fermionic lattice systems with power-law interactions at non-zero temperature, Phys. Rev. Lett. 119, 
110601 (2017), arXiv: 1702.00371 [quant-ph]

[31] L. Lepori, D. Vodola, G. Pupilo, G. Gori, A. Trombettoni, Effective Theory and Breakdown of Conformal Symmetry in a Long-Range Quantum Chain, Annals of Physics 374, 35-66 (2016), arXiv:1511.05544 [cond-mat.str-el]

[32] M. van Regemortel, D. Sels, M. Wouters, Information propagation and equilibration in long-range Kitaev chains, Phys. Rev. A, 93, 032311 (2016), arXiv:1511.05459 [cond-mat.stat-mech]

[33] L. Lepori, A. Trombettoni, D. Vodola, Singular dynamics and emergence of nonlocality in long-range quantum models, J. Stat. Mech. 033102 (2017), arXiv:1607.05358 [cond-mat.str-el]

[34] K. Patrick, T. Neupert, J. K. Pachos, Topological Quantum Liquids with Long-Range Couplings, Phys. Rev. Lett. 118, 267002 (2017), arXiv:1611.00796 [cond-mat.str-el]

[35] L. Lepori, L. Dell'Anna, Long-range topological insulators and weakened bulk-boundary correspondence, New. J. Phys. 19, 103030 (2017), arXiv:1612.08155 [cond-mat.str-el]

[36] A. Alecce, L. Dell'Anna, Extended Kitaev chain with longer-range hopping and pairing, Phys. Rev. B 95, 195160 (2017), arXiv:1703.10086 [cond-mat.str-el]

[37] O. Viyuela, D. Vodola, G. Pupillo, M. A. Martín-Delgado, Topological Massive Dirac Edge Modes and Long-Range Superconducting Hamiltonians, Phys. Rev. B 94, 125121 (2016), arXiv: 1511.05018 [cond-mat.str-el]

[38] P. Cats, A. Quelle, O. Viyuela, M. A. Martín-Delgado, C. Morais-Smith, Staircase to Higher Topological Phase Transitions, Phys. Rev. B 97, 121106 (2018), arXiv: 1710.05691 [cond-mat.stat-mech]

[39] N. Sedlmayr, P. Jäger, M. Maiti, J. Sirker, A bulk-boundary correspondence for dynamical phase transitions in one-dimensional topological insulators and superconductors, Phys. Rev. B 97, 064304 (2018), arXiv:1712.03618 [cond-mat.stat-mech]

[40] NIST Digital Library of Mathematical Functions, http://dlmf.nist.gov/, Release 1.0.18 of 2018-03-27. F. W. J. Olver, A. B. Olde Daalhuis, D. W. Lozier, B. I. Schneider, R. F. Boisvert, C. W. Clark, B. R. Miller, and B. V. Saunders, eds.

[41] B.-Q. Jin, V.E. Korepin, Quantum Spin Chain, Toeplitz Determinants and Fisher-Hartwig Conjecture, J. Stat. Phys. 116 (2004) 157-190, arXiv:quant-ph/0304108

[42] A. R. Its, B. Q. Jin, V. E. Korepin, Entropy of XY spin chain and block Toeplitz deter- 
minants, Fields Institute Communications, Universality and Renormalization, vol 50, page 151, (2007), arXiv:quant-ph/06066178

[43] A. R. Its, F. Mezzadri, M. Y. Mo, Entanglement entropy in quantum spin chains with finite range interaction, Comm. Math. Phys. Vo. 284 117-185 (2008), arXiv:0708.0161v2 [math-ph]

[44] Z. Kádar, Z. Zimborás, Entanglement entropy in quantum spin chains with broken reflection symmetry, Phys. Rev. A 82, 032334 (2010), arXiv:1004.3112 [quant-ph]

[45] M. Kormos, Z. Zimborás, Temperature driven quenches in the Ising model: appearance of negative Rényi mutual information, J. Phys. A: Math. Theor. 50264005 (2017), arXiv:1612.04837 [cond-mat.stat-mech]

[46] O. Toeplitz, Zur Transformation der Scharen bilinearer Formen von unendlichvielen Veränderlichen, Nachr. der Kgl. Gessellschaft der Wissen schaften zu Göttingen, Mathematisch-physikalische Klasse (1907), 110-115, and Zur Theorie der quadratischen und bilinearen Formen von unen dlichvielen Veränderlichen. I. Teil: Theorie der L-Formen, Math. Ann. 70, (1911), 351376

[47] G. Szegö, Collected papers, Vol. 1, 19151927, edited by R. Askey, Birkhuser, 1982.

[48] G. Szegö, Ein Grenzwertsatz über die Toeplitzschen Determinanten e iner reellen positiven Funktion, Math Ann. 76 (1915), 490-503.

[49] P. Deift, A. Its, I. Krasovsky, Toeplitz matrices and Toeplitz determinants under the impetus of the Ising model. Some history and some recent results, Comm. Pure Appl. Math. 66 (2013), 1360-1438, arXiv:1207.4990v3 [math.FA]

[50] B. Kaufman, L. Onsager, Crystal Statistics, III. Short-Range Order in a Binary Ising Lattice, Phys. Rev. 761244 (1949)

[51] G. Szegö, On certain hermitian forms associated with the Fourier series of a positive function, Festschrift Marcel Riesz, Lund (1952), 228238

[52] I. A. Ibraginov, A theorem of Gabor Szegö, Mat. Zametki 3 (1968), 693702 (In Russian, English translation: Math. Notes 3 (1968), 6, 442448)

[53] A. Lenard, Momentum Distribution in the Ground State of the One-Dimensional System of Impenetrable Bosons, J. Math. Phys. 5 (7) (1964), 930943

[54] T. T. Wu, Theory of Toeplitz Determinants and the Spin Correlations of the TwoDimensional Ising Model. I, Phys. Rev. 149 (1966), 380400 
[55] M. E. Fisher, R. E. Hartwig, Toeplitz determinants, some applications, theorems and conjectures, Adv. Chem. Phys. 15, 333-353 (1968)

[56] E. L. Basor, A localization theorem for Toeplitz determinants, Indiana Math. J. 28, 975 (1979)

[57] B. Gyires, Eigenwerte verallgemeinerter Toeplitzschen Matrizen, Publ. Math. Debrecen 4 (1956), 171179.

[58] I. I. Hirschman, Matrix-valued Toeplitz operators, Duke Math. J. 34, (1967), 403-415

[59] H. Widom Toeplitz Determinants with Singular Generating Functions, Am. Journal of Math. 95(1973), pp. 333-383

[60] H. Widom, Asymptotic Behavior of Block Toeplitz Matrices and Determinants II, Adv. in Math. 21:1 (1976), 129

[61] H. Widom, On the Limit of Block Toeplitz Determinants, Proceedings of the American Mathematical Society, Volume 50, 1, 167-173 (1975)

[62] Note that near these points the energy behaves linearly with $\left|\theta-\theta_{F}\right|$, as in a massless relativistic dispersion relation.

[63] A matrix is of Cauchy type if its entries are of the form $C_{n m}=\left(X_{n}-Y_{m}\right)^{-1}$ with $X_{n}-Y_{m} \neq$ 0 , and $X_{n}, Y_{m} \in \mathbb{C}$ where $n, m \in \mathbb{N}$.

[64] The log derivative in (24) for the linear term produces simple poles at $\lambda= \pm 1$ and since $f_{\alpha}( \pm 1)=0$ the integral vanishes 